\title{
A NOTE ON THE EARTH-STRETCHING APPROXIMATION FOR LOVE WAVES
}

\author{
By David P. Hill and Don L. Anderson
}

Earth-flattening transformations provide an efficient means for computing Lovewave dispersion and torsional normal mode frequencies in radially heterogeneous, spherically symmetric earth models. These transformations involve simple algebraic scaling factors applied to solutions for $S H$ waves in a layered half-space.They result in considerable computational savings over solutions expressed directly in spherical geometry. Several earth-flattening transformations for $S H$ waves are described in the literature (Anderson and Toksöz, 1973; Sato, 1968; Biswas and Knopoff, 1970). Chapman (1973) has examined the general class of power-law earth-flattening transformations and their application to body-wave problems.

In this note we clarify two points regarding the earth-stretching approximation used by Anderson and Toksöz (1963). In their notation, the transformation between spherical and fat variables for the approximation is

$$
\begin{aligned}
r & =a-h \\
V(r) & =(a / r) \chi(h)
\end{aligned}
$$

where $r$ is the radial variable, $a$ is a reference radius, $h$ is the depth variable (positive downward), and $V(r)$ and $\chi(h)$ are the spherical and flat transverse displacements, respectively. (The original transformation (1b) was written without the factor, $a$, which is dimensionally incorrect.) Here, we recognize equation (1a) as an approximation to the exact depth transformation

$$
r=a \exp (-h / a) ; h=-a \ln (r / a)
$$

for $|h / a| \ll 1$ (see Biswas and Knopoff, 1970; Muller, 1971; or Hill, 1972).

A previously unresolved problem has involved proper justification of the form of the stress component appearing in boundary conditions under this transformation. In spherical geometry, the appropriate stress coninuity condition across a boundary at $r=a$ is

$$
\left\{\mu\left[\frac{d}{d r} V(r)-\frac{V(r)}{r}\right]\right\}_{r=a_{+}}=\left\{\mu\left[\frac{d}{d r} V(r)-\frac{V(r)}{r}\right]\right\}_{r=a_{-}} .
$$

Substituting (1) into (3), and noting the necessity for implicit differentiation gives

$$
\left[\frac{d}{d h}\left(\frac{\chi(h)}{a-h}\right) \frac{d h}{d r}-\frac{\chi(h)}{(a-h)^{2}}\right]
$$

for the terms in the brackets. Evaluation leads directly to

$$
\left\{\mu\left[\frac{d}{d h} \chi(h)+\frac{2 \chi(h)}{a}\right]\right\}_{h=0_{-}}=\left\{\mu\left[\frac{d}{d h} \chi(h)+\frac{2 \chi(h)}{a}\right]\right\}_{h=0_{+}} .
$$

This is the correct form of the stress condition in "flat" geometry under transformation (1), and it is the form that was used but not derived by Anderson and Toksöz (1963). Putting the exact depth transformation (2) in (3) with $V=(a / r) \chi$ leads to the same result. 
The natural stress condition for $S H$ waves across a plane boundary is

$$
\left\{\mu \frac{d}{d z} \chi(z)\right\}_{z=0_{+}}=\left\{\mu \frac{d}{d z} \chi(z)\right\}_{z=0_{-}} .
$$

Thus the additional term $2 \chi / a$ from (4) must be included with the stress conditions in computing the Thompson-Haskell matrices for layered half-space programs used with this earth-stretching approximation. The transformation between spherical and flat $S H$ displacements (or potentials) used by Biswas and Knopoff (1970) and Chap$\operatorname{man}(1973)$ is

$$
V(r)=(r / a) \chi(h) .
$$

This choice results in a flat stress condition with the same form as the natural stress condition for a plane boundary. Their transformation thus has the advantage that the layered half-space Thompson-Haskell matrices can be used directly in transformed spherical problems. The modification required in layered half-space programs with the Anderson-Toksöz method, however, is of a trivial nature.

Finally, the correct relation between the wave number of traveling waves, $k$, and the order number of standing waves, $n$, in a spherical body is

$$
n(n+1)=k^{2} a^{2}-\frac{1}{4} .
$$

Anderson and Toksöz (1963) omitted the quantity $\frac{1}{4}$ in the torsional equation of motion for a spherical shell (equation 1 in their paper). Including this term changes $k^{2}$ in their equations $(5),(6)$, and $(7)$ to $\left(a^{2} k^{2}-\frac{1}{4}\right) / a^{2}$. The absence of the quantity $\frac{1}{4}$, however, only becomes significant for very low frequencies or order numbers.

\section{ACKNOWLEDGMENTS}

We have profited from helpful discussions with David G. Harkrider. This research was partially supported by the Advanced Research Projects Agency of the Department of Defense and was monitored by the Air Force Office of Scientific Research under Contract F44620-72-C-0078.

\section{REFERENCES}

Anderson, D. L. and M. N. Toksöz (1963). Surface waves on a spherical earth; 1. Upper mantle structure from Love waves, $J$. Geophys. Res. 68, 3483-3500.

Biswas, N. N. and L. Knopoff (1970). Exact earth-flattening calculation for Love waves, Bull. Seism. Soc. Am 60, 1123-1137.

Chapman, C. H. (1973). The earth-ffattening transformation in body-wave theory, Geophys. $J$. $35,55-70$.

Hill, D. P. (1972). An earth-flattening transformation for waves from a point source. Bull. Seism. Soc. Am. 62, 1195-1210.

Muller, G. (1971). Approximate treatment of elastic body waves in media with spherical symmetry, Geophys. J. 32, 435-449.

Sato, R. (1968). Effect of spherical crustal layering (SH waves), J. Phys. Earth 16, 1-6.

U. S. Geological Survey

Menlo Park, California 94025 (D.P.H.)

Division of Geologitcal and Planetary Sciences

California Institute of Technology

Contribution No. 2779

Manuseript received August 9, 1976
Seismological Laboratory

California Institute of Technology

Pasadena, California 91125 (D.L.A.) 